\title{
Health-related quality of life after fast-track treatment results from a randomized controlled clinical equivalence trial
}

\author{
Ghislaine A. P. G. van Mastrigt • Manuela A. Joore • \\ Fred H. M. Nieman • Johan L. Severens • \\ Jos G. Maessen
}

Accepted: 22 February 2010/Published online: 26 March 2010

(C) The Author(s) 2010. This article is published with open access at Springerlink.com

\begin{abstract}
Purpose This randomized clinical equivalence trial was designed to evaluate health-related quality of life (HRQoL) after fast-track treatment for low-risk coronary artery bypass (CABG) patients.

Methods Four hundred and ten CABG patients were randomly assigned to undergo either short-stay intensive care treatment (SSIC, $8 \mathrm{~h}$ of intensive care stay) or control treatment (care as usual, overnight intensive care stay). HRQoL was measured at baseline and 1 month, and one year after surgery using the multidimensional index of life quality (MILQ), the EQ-5D, the Beck Depression Inventory and the State-Trait Anxiety Inventory.

Results At one month after surgery, no statistically significant difference in overall HRQoL was found (MILQscore $P$-value $=.508$, overall MILQ-index $P$-value $=$ .543, EQ-5D VAS P-value $=.593$ ). The scores on the MILQ-domains, physical, and social functioning were significantly higher at one month postoperatively in the SSIC group compared to the control group $(P$-value $=$ $.049 ;$ 95\%CI: $0.01-2.50$ and $P$-value $=.014,95 \%$ CI:
\end{abstract}

G. A. P. G. van Mastrigt $(\bowtie) \cdot$ M. A. Joore ·

F. H. M. Nieman · J. L. Severens

Clinical Epidemiology and Medical Technology Assessment,

University Hospital Maastricht, Maastricht, Netherlands

e-mail: ghislainevanmastrigt@ orange.nl

M. A. Joore

e-mail:m.joore@mumc.nl

J. L. Severens

e-mail: h.severens@beoz.unimaas.nl

J. G. Maessen

Cardio Thoracic Surgery, University Hospital Maastricht,

Maastricht, Netherlands

e-mail: j.g.maessen@mumc.nl
0.24-2.06, respectively). However, these differences were no longer observed at long-term follow-up.

Conclusions According to our definition of clinical equivalence, the HRQoL of SSIC patients is similar to patients receiving care as usual. Since safety and the financial benefits of this intervention were demonstrated in a previously reported analysis, SSIC can be considered as an adequate fast-track intensive care treatment option for low-risk CABG patients.

Keywords Quality of life - Intensive care · Coronary artery bypass grafting .

Randomized controlled trial

$\begin{array}{ll}\text { Abbreviations } \\ \text { BDI } & \text { Beck depression inventory } \\ \text { CABG } & \text { Coronary artery bypass grafting } \\ \text { IC } & \text { Intensive care } \\ \text { MILQ } & \text { Multidimensional index of life quality } \\ \text { HRQoL } & \text { Health-related quality of life } \\ \text { SSIC } & \text { Short-stay intensive care } \\ \text { STAI-S } & \text { State anxiety scale } \\ \text { 95\% CI } & 95 \% \text { confidence interval }\end{array}$

\section{Introduction}

Rapid technological advancements changed the practice of intensive care medicine in the last few decades. These improvements facilitated the introduction of fast-track protocols for the treatment of cardiac surgical patients, which are very popular as they contribute to a more efficient utilization of the existing care facilities. Various 
reviews, based on randomized controlled studies, focused on the evaluation of the clinical effectiveness of different types of fast-track treatments [1-3].

However, for the evaluation of these intensive care interventions it is essential to assess its impact on healthrelated quality of life [4]. These patient-reported outcomes contain valuable information for intensive care physicians, other healthcare providers, policymakers, patients, and their relatives.

Several fast-track trials incorporated HRQoL as an outcome measure in their evaluations, but only one specific domain with a short follow-up time has been assessed [5-17]. These limited assessments are helpful in describing the domains of patient outcome in a specific area of HRQoL. However, they are not designed to evaluate either the overall health state [18] or all domains of QoL that may contain important aspects of health. For a comprehensive evaluation of HRQoL, it is important to assess both overall or generic HRQoL and disease-specific HRQoL [19]. We chose to use the EQ-5D, as a generic measure, because it is brief, simple, and easy to administer. The reliability and validity for the EQ-5D has been studied in cardiac patients $[20,21]$ and in critical care patients [22]. In a previous paper, we also reported generic HRQoL at 1 month after surgery as an outcome measure in the economic evaluation of the short-stay intensive care treatment [23]. The patient's answers to the five EQ-5D items were used to calculate the utility value (a score of $0-1$ ), based on population weights [24]. In the current study, this outcome was not reported because the aim of this previous paper was to report data from a patient's point of view and not from a societal perspective. As a disease-specific measure, the multidimensional index of life quality (MILQ) was chosen. Although this questionnaire is not frequently used, its reliability and validity has been tested by Avis et al. [25] for cardio vascular patients. In addition, we were interested in assessing the impact of the fast-track treatment on anxiety and depression, as these psychological aspects of HRQoL may be affected after cardiac surgery [26].

As several studies [22, 27-29] on intensive care admission have demonstrated the importance of long-term follow-up, in addition to 1-month follow-up, we assessed the impact of the fast-track treatment on HRQoL at 1 year after surgery.

The focus of this randomized clinical equivalence trial is to evaluate the impact of a fast -track protocol, i.e., early discharge -within 8-h of postsurgical coronary artery bypass patients from intensive care to medium care (MC) on disease-specific, generic, and domain-specific (anxious and depressive feelings) HRQoL up to 1 year postoperatively.

\section{Materials and methods}

Study design and hypothesis

This single blinded randomized clinical equivalence trial was designed to evaluate the HRQoL of low-risk CABG patients at 1 year postoperatively who were randomized for either short-stay intensive care (SSIC $<8 \mathrm{~h}$ ) or overnight intensive care stay (control group patients). The choice of the study design was based on the expected therapeutic equivalence of the SSIC treatment and the care as usual. We assumed there would be fewer costs in the SSIC group but comparable clinical effectiveness and QoL outcomes in both trial arms. The clinical- and cost-effectiveness outcomes of the SSIC trial are reported elsewhere [23].

The following hypothesis was formulated to evaluate the primary outcome:

At one-year postoperatively, the disease-specific HRQoL (measured by means of MILQ) will be similar in both groups.

Two additional hypotheses were formulated to assess the secondary outcome measures:

At one year after surgery, both generic HRQoL (measured by means of EQ-5D) and domain-specific HRQoL (measured by means of Beck Depression Inventory and Spielberger State Anxiety Scale) will be similar in both groups.

At one month postoperatively, disease-specific, generic, and domain-specific HRQoL will also be similar for both groups.

\section{Patients}

All CABG patients who were admitted to the University Hospital of Maastricht in the period between February 2001 and March 2003 were considered for inclusion. High-risk $\mathrm{CABG}$ patients were separated from the low-risk patients by means of a risk stratification system, based on the criteria of Parsonnet score [30] and the Society of Thoracic Surgeons [31]. The exclusion criteria were defined as follows: age older than 78 , ejection fraction of less than $30 \%$, stage 3 obesity (BMI $>40 \mathrm{~kg} / \mathrm{m}^{2}$ ), hemodialysis (kidney-replacing therapy), pulmonary hypertension (systolic pressure $<40 \mathrm{mmHg}$ ), recent cardiovascular accident $(<1$ month), recent myocardial infarction $(<24 \mathrm{~h})$, cardiogenic shock (systolic blood pressure $<80 \mathrm{mmHg}$, -central filling pressure of $>20 \mathrm{mmHg}$, -cardiac index of $<1.8 \mathrm{Lmin}^{-1} \mathrm{~m}^{-2}$ ), or the need for inotropic therapy $\left(>5 \mathrm{mg} / \mathrm{\mu g}^{-1} \mathrm{~min}^{-1} /\right.$ dopamine or dobutamine), ongoing infarction (a significant increase in myocardial muscle creatine kinase isoenzyme MB within $4 \mathrm{~h}$ before surgery), or the need for intra-aortic balloon pump. Other reasons for exclusion were, for instance, 
inability to give informed consent, inability to speak/read/ understand the Dutch language and emergency surgery. The local ethical and research council approved the study.

\section{Randomization and blinding}

Before starting the study, a list of randomly permutated blocks (with a length of 10) were generated for either "control" $(n=300)$ or "SSIC" $(n=300)$ by a computer, and the results were entered sequentially into numbered, opaque envelopes, sealed with tape. The envelopes were opened by IC nurses immediately upon IC admission. Patients and the surgical team were blinded to the group assignments.

\section{Treatment}

Until IC admission, treatment was similar for both groups. In the IC, both groups received standard IC monitoring. In order to allow the transfer of SSIC patients from IC to MC within $8 \mathrm{~h}$, the discharge criteria described in Table $1 \mathrm{had}$ to be fulfilled. The flow chart (Fig. 1) shows the timelines for both groups. The SSIC patients who did not fulfill the

Table 1 Description of criteria for discharge from IC for both control and short-stay intensive care (SSIC) patients

\footnotetext{
Pulmonary

Patient needs to be extubated for at least $30 \mathrm{~min}$ before he/she can be transferred to the $\mathrm{MC}^{\mathrm{a}}$

Breathing frequency $\geq 10 \leq 25$ times per minute

Arterial blood gas $\mathrm{pO}_{2}>10 \mathrm{kPa}^{\mathrm{b}}$ and the $\mathrm{PCO}_{2}^{\mathrm{c}}<6.0 \mathrm{Kpa}$, depending on clinical glance can be deviated form this value (in consultation with ward doctor)

Cardiac

Patient has no ongoing infarction (a significant increase in enzyme "CKMB" ) or ischemic changes on the $\mathrm{ECG}^{\mathrm{e}}$ or arrhythmia with hemodynamic consequences

Fluid balance

Patient has $\leq 100 \mathrm{ml}$ chest tube drainage per hour and a diuresis $>0.5 \mathrm{ml} / \mathrm{kg} / \mathrm{hour}$

Neurological

Patient has no signs/symptoms of neurological complications

Hemodynamic and supporting therapy

Patient does not need inotropic support or sodium nitroprusside ( $\mathrm{NTG}^{\mathrm{f}}$ and dopamine $(2 \mathrm{mcg} / \mathrm{kg} / \mathrm{min})$ are allowed)

Patient does not have an intra-aortic balloon pump

Patient with pulmonary artery catheter: the last measured cardiac index $\geq 2 \mathrm{~L} / \mathrm{min} / \mathrm{m}^{2}$ or mixed venous saturation $(55-60 \%$ )

${ }^{\text {a } M C \text { Medium Care }}$

b Kpa Kilo Pascal

c $\mathrm{PCO}_{2}$ Partial Pressure of Carbon Dioxide

${ }^{\mathrm{d}} C K M B$ Creatine Kinase $\mathrm{Mb}$

e $E C G$ Electrocardiogram

${ }^{\mathrm{f}} N T G$ Nitroglycerine
}

discharge criteria within $8 \mathrm{~h}$ and all control group patients were treated according to usual care.

For logistical reasons, it was not possible to transfer SSIC patients who fulfilled the criteria for discharge within $8 \mathrm{~h}$ from the IC to the MC situated on another floor in the hospital. Consequently, the nurse/patient ratio of one nurse to one patient was maintained. However, the care provided by the nurse was adapted to the MC guidelines. In the morning after surgery, all patients were transferred to a MC ward connected to the general ward. The nurse/patient ratio in this MC was one nurse to two patients. Patients stayed there for another $24 \mathrm{~h}$.

On the second postoperative day, patients were admitted to the general ward, where they stayed until discharge or transfer to the district hospital.

\section{Procedures HRQoL measurement}

After obtaining informed consent, HRQoL questionnaires were self-administered by the patients the day before surgery at the hospital, at 1-month and one-year postoperatively at home. If a patient was unable to complete the questionnaire individually, the research nurse performed an interviewed administration. The application of the four HRQoL instruments took almost $20 \mathrm{~min}$ and was done in a fixed order, first the EQ-5D, secondly the MILQ, followed by Spielberger State-Trait Anxiety Inventory (STAI-S) and Beck Depression Inventory (BDI). Patients who did not respond to the follow-up questionnaires received a written reminder. If the patients still did not respond, they were contacted by telephone to find out the reasons for non-response.

HRQoL questionnaires

The multidimensional index of life quality (MILQ), a HRQoL measure specially developed for cardiovascular disease, was used to measure disease-specific QoL [25]. In the first item (which is referred to as the "MILQ-score"), the respondent is asked to rate his/her general HRQoL on a seven-point scale (from $1=$ worst to $7=$ best possible HRQoL score). In the other 35 items, the respondent's degree of satisfaction with various aspects of quality of life is scored on a seven-point scale (from $1=$ very dissatisfied to $1=$ very satisfied). These items cover the following nine domains: mental health, physical health, physical functioning, cognitive functioning, social functioning, intimacy, financial status, relationship with health professionals, and productivity. As each domain contains four items, the total domain score can range from 4 to 28 (except for the domain of productivity which contains three items, the total score range from 3 to 21). The Dutch translation of the MILQ has been used to assess the HRQoL of CABG-patients [32]. An unweighted index was calculated by adding up over all nine-domain scores, will possible scores 


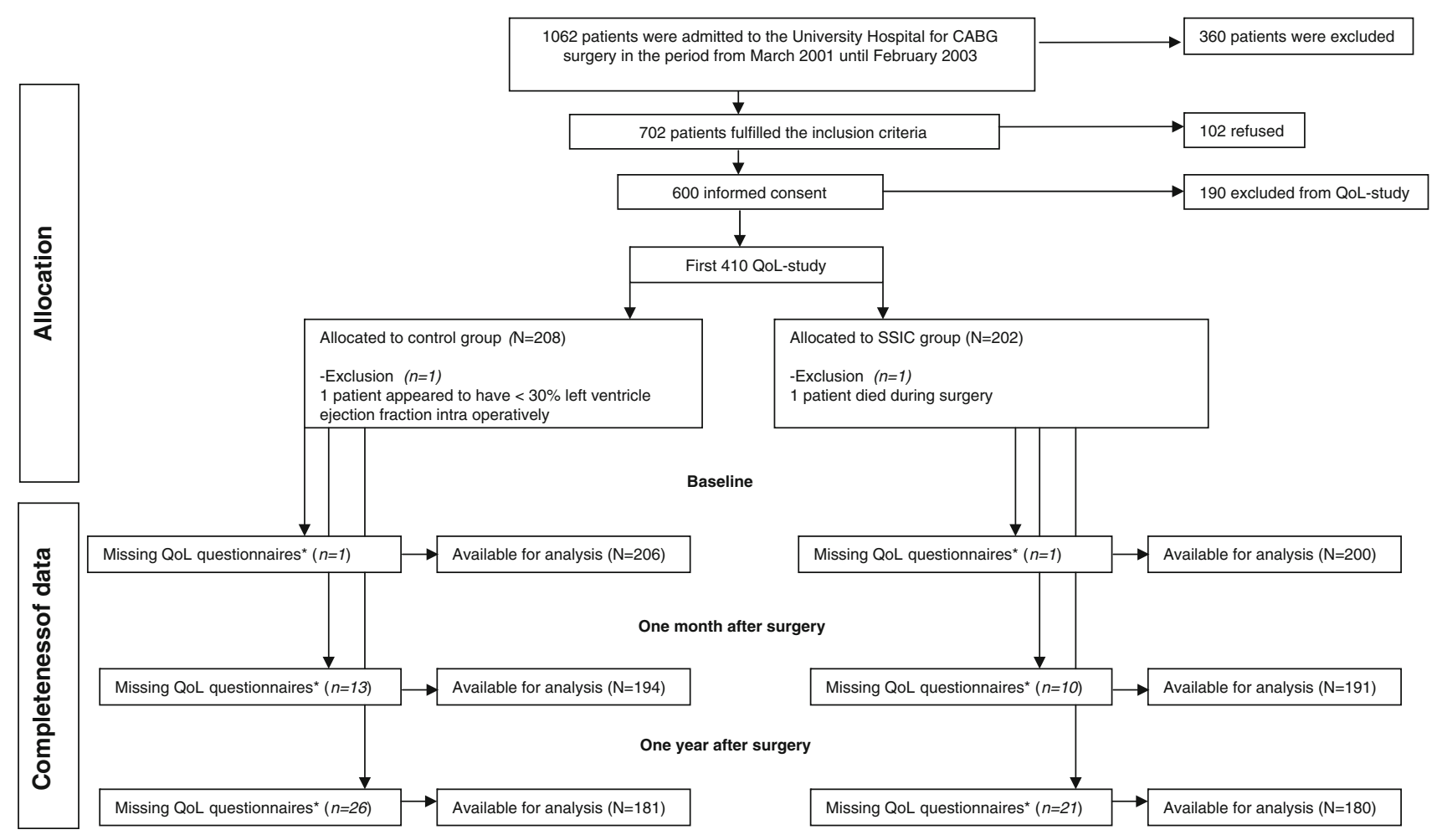

Fig. 1 Flow chart of patient inclusion (* for reasons see Table 3)

range from 35 to 245 (which is referred to as the "overall MILQ-index").

Furthermore, generic HRQoL was measured by the EQ5D [33]. The EQ-5D consists of two components: an EQ-5D descriptive system and an EQ-5D Visual Analogue Scale (EQ-5D VAS). In the descriptive system, the respondent is asked to rate his or her health by checking one of three levels of severity - "no problems" (coded as 1), "some or moderate problems" (coded as 2), "severe problems or unable to perform" (coded as 3)- in each of the following 5 EQ-5D dimensions: mobility, self-care and pain/discomfort, usual activities, and anxiety/depression. For the EQ-VAS, participants draw a line from a box to the point on the thermometer-like scale corresponding to their health state (with a range from 0 "the worst imaginable health state" to 100 "the best imaginable health state"). Depression was evaluated by means of BDI [34]. The STAI-S was used to measure temporal and transient aspects of anxiety [35].

Both STAI-S (20 items) and BDI (21 items) contain items expressed as four-point scales. Both BDI and STAI have been used in studies with CABG patients [36-38] and are considered to be validated and reliable instruments $[34,35]$.

One-year quality of life equivalence and power analysis

Equivalence in health-related HRQoL was defined a priori. It was expected that the disease-specific HRQoL (MILQ) would measure the most sensitive to detect differences between the groups. The overall MILQ-index varies between 1 (worst of health) and 7 (best of health). Equivalence on the overall MILQ-index at 1 year was assumed if the SSIC and the control group would not differ within a tolerance range of $5 \%$, i.e., within 0.4 on a seven-point scale. It was assumed that the standard deviation of this difference would be 1.15 [32]. Based on this, so with a twosided test, alpha of 0.05 , a power of $90 \%$, and an expected drop-out percentage of $10 \%$, the number of patients needed amounted to 194 for each study group. For the EQ-5D domains and EQ-5D VAS, the power calculations determined a difference of 0.3 points on the EQ-5D domains and a difference of 8 points on the EQ-5D VAS could be detected with $90 \%$ power.

Statistical analysis

The reasons for non-response were categorized as follows: "Informative censoring" (if reason was non-random e.g., related to the health status) [39], "non-informative censoring" (if reason was random) [39], and "unclear" (if reason was unknown).

All analyses were conducted following the intention-totreat principle. Missing values were imputed by the separate mean group scores of non-missing patients for the SSIC and the control groups. We performed the analysis with and without mean imputation, and we did not find a difference in study findings. 
HRQoL scores at baseline were reported in means and standard deviations and at follow-up as mean change scores (baseline minus 1 month or 1 year minus 1 month) with a $95 \%$ confidence interval $(95 \% \mathrm{CI})$ for both groups. The Kolmogorov-Smirnov test was used to test for normality of differences between postoperative and preoperative scores. Student t-tests were performed to examine the mean differences in HRQoL change between both treatment groups at 1 month after surgery.

Repeated measures ANCOVA using the preoperative scores as a covariate was performed to test equivalence of HRQoL scores at 1 year. Interaction effects between preoperative HRQoL and the treatment modality group on one-year differences in HRQoL were also tested for statistical significance. Next, in regression analyses with the MILQ-score, the overall MILQ-index or the EQ-5D VAS as dependent variables the relationships with other factors were tested. Selection of the potential predictors in the regression models was based on results of previous research (see appendix). Regression modeling was done with list wise deletion of missing cases using both forward selection and backward elimination techniques. Predictors statistically significant in both techniques were selected in the model, except for treatment modality group, which was always kept within the model, irrespective of its statistical significance. Final regression model results are presented in a table with unstandardized effects, $P$-values and variance explained ( $R$-square) by each final model.

A $P$-Value of less than 0.05 was assumed to be statistically significant. All analysis was performed using SPSSpc version 12.0.1 (SPSS, Chicago, IL).

\section{Results}

Study population

Between February 2001 and March 2003, 702 out of the 1062 available CABG patients were eligible for inclusion in the clinical part of the study (Fig. 1). They were asked to give informed consent. A total of 600 patients gave informed consent and participated in the SSIC trial. For this QoL-study, only the first 410 of the included CABG patients were needed.

The study population consisted of a group of low-risk CABG patients with a mean age of 62 years, and $80 \%$ of them were men (Table 2).

The non-response rates were low and appear to be similar for control and SSIC groups in all three categories (informative, non-informative, and unclear) and at the three measurement times (Table 3). Four patients died (control $n=1$ and SSIC $n=3$ ) and one patient was readmitted to hospital. These events were unrelated to the IC treatment received. After ruling out mortality, 39 follow-up questionnaires $(4.8 \%$ of the total) were not sent back for reasons that were unclear.

The non-responders were mainly women with no partner who had slightly longer surgery times and hospital stays compared to the responders. However, there was no difference in patient characteristics between the nonresponders in the control group and the non-responders in the SSIC group (data not shown).

Baseline HRQoL outcomes appeared not to be significantly different between SSIC and control group ( $P$-Values not shown).

Disease-specific health-related quality of life

At 1 month after surgery, the change scores (1 month postoperative minus baseline) of both the MILQ-score and overall MILQ-index were not significantly different between the two treatment groups (MILQ-score $P$-Value $=.270$; 95\% CI: $-0.11-0.40$, overall MILQ-index $P$-Value $=.154$, 95\% CI: -1.49 to -9.41 ) (Table 4). However, the domains' physical and social functioning of the MILQ were significantly better in the SSIC group compared to the control group (respectively, $P$-Value $=.049,95 \% \mathrm{CI}$ : $0.01-2.50$ and $P$-Value $=.014,95 \%$ CI: 0.24-2.06) (Table 4).

Results of the repeated measures ANCOVA analysis with the baseline QoL scores as covariates show no statistically significant differences between the SSIC group and the control group in the linear trend in the year after surgery on the MILQ-score $(P$-Value $=.508)$, nor on the overall MILQ-index $(P$-Value $=.543)($ Table 4$)$.

In the regression models, the linear trend of the MILQscore and the linear trend of the overall MILQ-index were used as dependent variables. Controlled for potential confounding factors, the type of intensive care treatment received was again not a significant predictor of $\mathrm{HRQOL}$ (MILQ-score $P$-Value $=.736$, 95\% CI: $-.115-.162$, nor for the overall MILQ-index $P$-Value $=.934,95 \% \mathrm{CI}$ : -3.64-3.35) (Table 5).

Apart from this, the baseline scores of the corresponding QoL measures were significant predictors of linear trend of QoL. All tests for first-order interactions, and all tests on the other predictors like gender, age, and disease severity (appendix), were not statistically significant (Table 5).

Generic health-related quality of life

The change scores of the EQ-5D VAS and the five separate EQ-5D domains were not significantly different for both groups at one month after surgery (Table 4). HRQoL increased similarly in both groups in the first year after surgery on EQ-5D VAS $(P$-Value $=.593)$ and on all five EQ-5D domains (Table 4). 
Table 2 Comparison of demographic data, previous medical and cardiac history, and operative data between control $(N=207)$ and short-stay intensive care (SSIC) patients $(N=201)$

\begin{tabular}{|c|c|c|c|c|c|}
\hline & & \multicolumn{4}{|c|}{ Study population } \\
\hline & & $N^{\mathrm{a}}$ & Control & $N^{\mathrm{a}}$ & SSIC \\
\hline \multicolumn{6}{|l|}{ Demographic data } \\
\hline Age (Years) & Mean $\pm \mathrm{SD}^{\mathrm{b}}$ & 206 & $62.0 \pm 13.77$ & 201 & $61.8 \pm 10.67$ \\
\hline Body mass index $\left(\mathrm{Kg} / \mathrm{M}^{2}\right)$ & Mean \pm SD & 193 & $27.4 \pm 3.84$ & 185 & $27.8 \pm 3.55$ \\
\hline Sex (male) & $n(\%)$ & 207 & $169(81.6)$ & 201 & $161(80.1)$ \\
\hline Civil state & & 201 & & 198 & \\
\hline Married & $n(\%)$ & & $150(72.5)$ & & 158 (78.6) \\
\hline Unmarried no partner & $n(\%)$ & & $40(19.3)$ & & $31(15.4)$ \\
\hline Unmarried with partner & $n(\%)$ & & $14(6.8)$ & & $9(4.5)$ \\
\hline Education & & 195 & & 196 & \\
\hline Primary school not finished & $n(\%)$ & & $2(1.0)$ & & $5(2.5)$ \\
\hline Primary school & $n(\%)$ & & $53(25.6)$ & & $40(19.9)$ \\
\hline Lower education & $n(\%)$ & & $47(22.7)$ & & $57(28.4)$ \\
\hline Intermediate education & $n(\%)$ & & $63(30.4)$ & & $64(31.8)$ \\
\hline High education & $n(\%)$ & & $30(14.5)$ & & $30(14.9)$ \\
\hline \multicolumn{6}{|l|}{ Previous cardiac and medical history } \\
\hline Left ventricular ejection fraction & $n(\%)$ & 193 & & 189 & \\
\hline$>35 \%$ & & & $115(55.6)$ & & $120(59.7)$ \\
\hline $35-45 \%$ & & & $30(14.5)$ & & $27(13.4)$ \\
\hline $40-50 \%$ & & & $48(23.2)$ & & $42(20.9)$ \\
\hline NYHA classification angina ${ }^{c}$ & $n(\%)$ & 195 & & 191 & \\
\hline I & & & $8(3.9)$ & & $5(2.5)$ \\
\hline II & & & $41(19.8)$ & & $26(12.9)$ \\
\hline III & & & $82(39.6)$ & & $96(47.8)$ \\
\hline IV & & & $64(30.9)$ & & $64(31.8)$ \\
\hline Hypertension & $n(\%)$ & 204 & $88(42.5)$ & 201 & $86(42.8)$ \\
\hline Hypercholesterolemia & $n(\%)$ & 196 & $124(59.9)$ & 194 & $136(67.7)$ \\
\hline Smoking & $n(\%)$ & 199 & $69(33.3)$ & 198 & $73(36.3)$ \\
\hline Positive family anamneses & $n(\%)$ & 195 & $126(60.9)$ & 191 & $117(58.2)$ \\
\hline Diabetes mellitus & $n(\%)$ & 205 & $38(18.4)$ & 201 & $33(16.4)$ \\
\hline \multicolumn{6}{|l|}{ Operative data } \\
\hline Number of grafts & Mean \pm SD & 204 & $3.2 \pm 1.3$ & 200 & $3.1 \pm 1.2$ \\
\hline Cardiopulmonary bypass time (in minutes) & Mean $\pm \mathrm{SD}$ & 151 & $81.6 \pm 35.3$ & 153 & $77.4 \pm 34.1$ \\
\hline Aorta cross-clamp time (in minutes) & Mean \pm SD & 151 & $52.5 \pm 25.9$ & 154 & $47.8 \pm 27.3$ \\
\hline Without aorta cross-clamping & $n(\%)$ & & $56(27.1)$ & & $47(23.4)$ \\
\hline Re-do CABG & $n(\%)$ & 207 & $14(6.8)$ & 201 & $10(5.0)$ \\
\hline \multicolumn{6}{|l|}{ Hospital stay } \\
\hline Intensive care stay & Mean \pm SD & 207 & $29.9 \pm 24.04$ & 201 & $19.8 \pm 27.30$ \\
\hline Total hospital stay & Mean \pm SD & 207 & $8.7 \pm 5.10$ & 201 & $8.0 \pm 4.41$ \\
\hline
\end{tabular}

${ }^{\text {a }} N$ number of patients with data available on the reported variable

b $S D$ Standard Deviation

${ }^{c}$ NYHA New York Heart Association

In the regression models, the type of intensive care treatment had no statistically significant effect on the linear trend of the EQ-5D VAS $(P$-Value $=.556,95 \% \mathrm{CI}:-2.81-$ 1.51; Table 5). The baseline scores of the EQ-5D VAS and baseline anxiety score and gender were significant predictors of the linear trend of the EQ-5D VAS. All tests for firstorder interactions, and all tests on the other predictors (appendix), were not statistically significant (Table 5). 
Table 3 Reasons of missing data in baseline, 1-month and 1 -year questionnaire in number (percentages)

a same patient

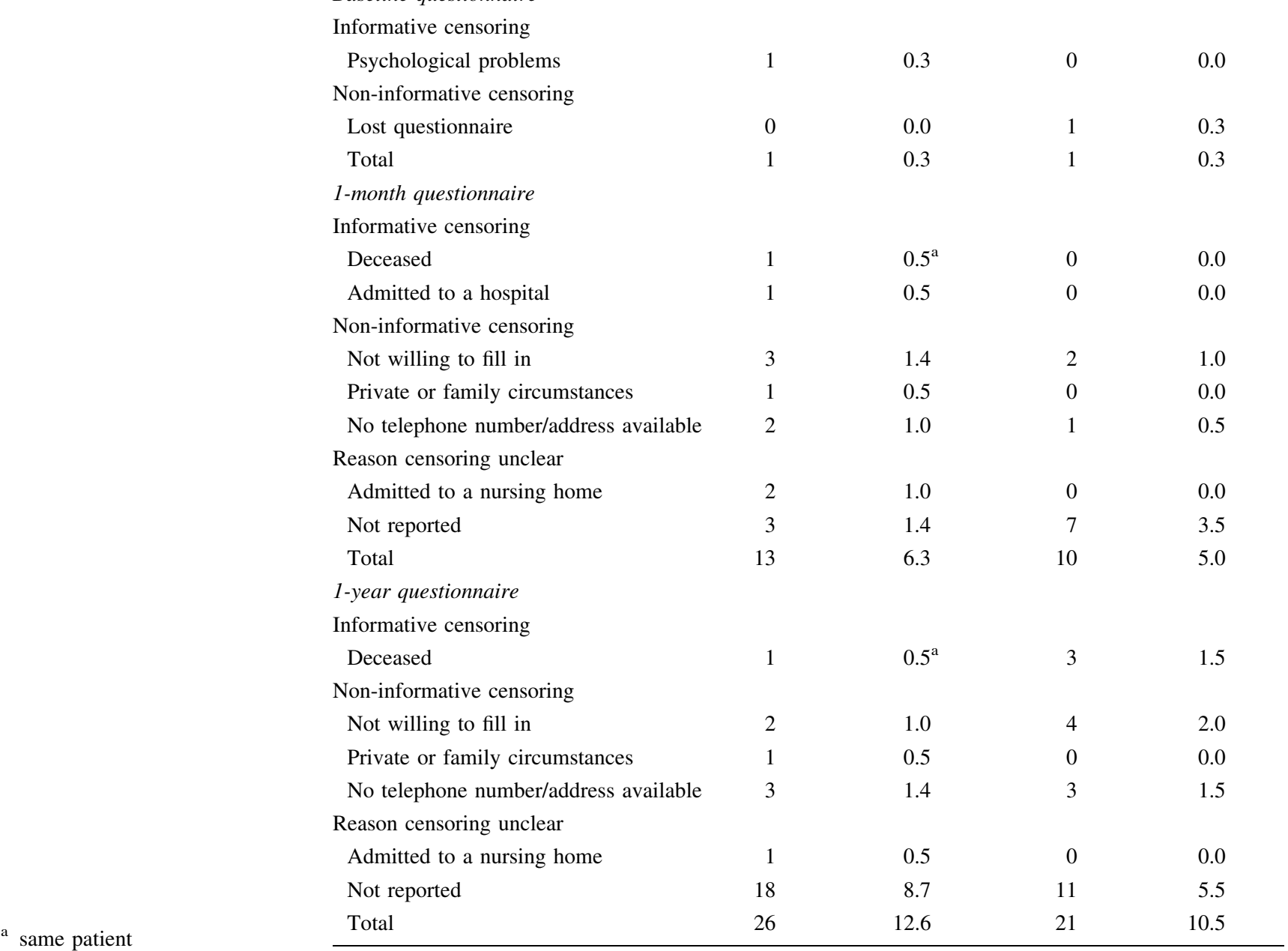

Domain-specific health-related quality of life

The change score of the BDI and STAI-S did not differ in both groups at 1 month after surgery $(P$-Value $=.496$, 95\% CI: $-0.13-0.65, P$-Value $=.259,95 \%$ confidence intervals: -3.8 to -1.03 ) (Table 4 ).

The results of the repeated measures ANCOVA analysis show no statistically significant differences in the linear trend at 1 year after surgery for both groups on BDI $(P$-Value $=.621)$ or STAI $-\mathrm{S}(P$-Value $=.653)($ Table 4$)$.

\section{Discussion}

Summary of study findings

This large, randomized controlled equivalence trial is to our knowledge the first study that evaluates the impact of a short- stay intensive care treatment (a type of fast-track treatment) on disease-specific, generic, and domain-specific HRQoL in the year after surgery in low-risk CABG patients.

After controlling for baseline differences and potential confounding factors, like gender and age, the changes of disease-specific, generic, and domain-specific HRQoL in the first year after surgery appear to be similar for patients who received the short-stay IC treatment (discharge within $8 \mathrm{~h}$ after surgery) and for those who stayed overnight on the IC. The scores on the domains' physical and social functioning of the MILQ were significantly higher in the SSIC group compared to the control group at 1 month after surgery. However, these differences were no longer observed at one-year follow-up.

Fast-track treatments and HRQoL

The desirability of decreasing postcardiac surgery intensive care utilization was recognized two decades ago [41], [40] 


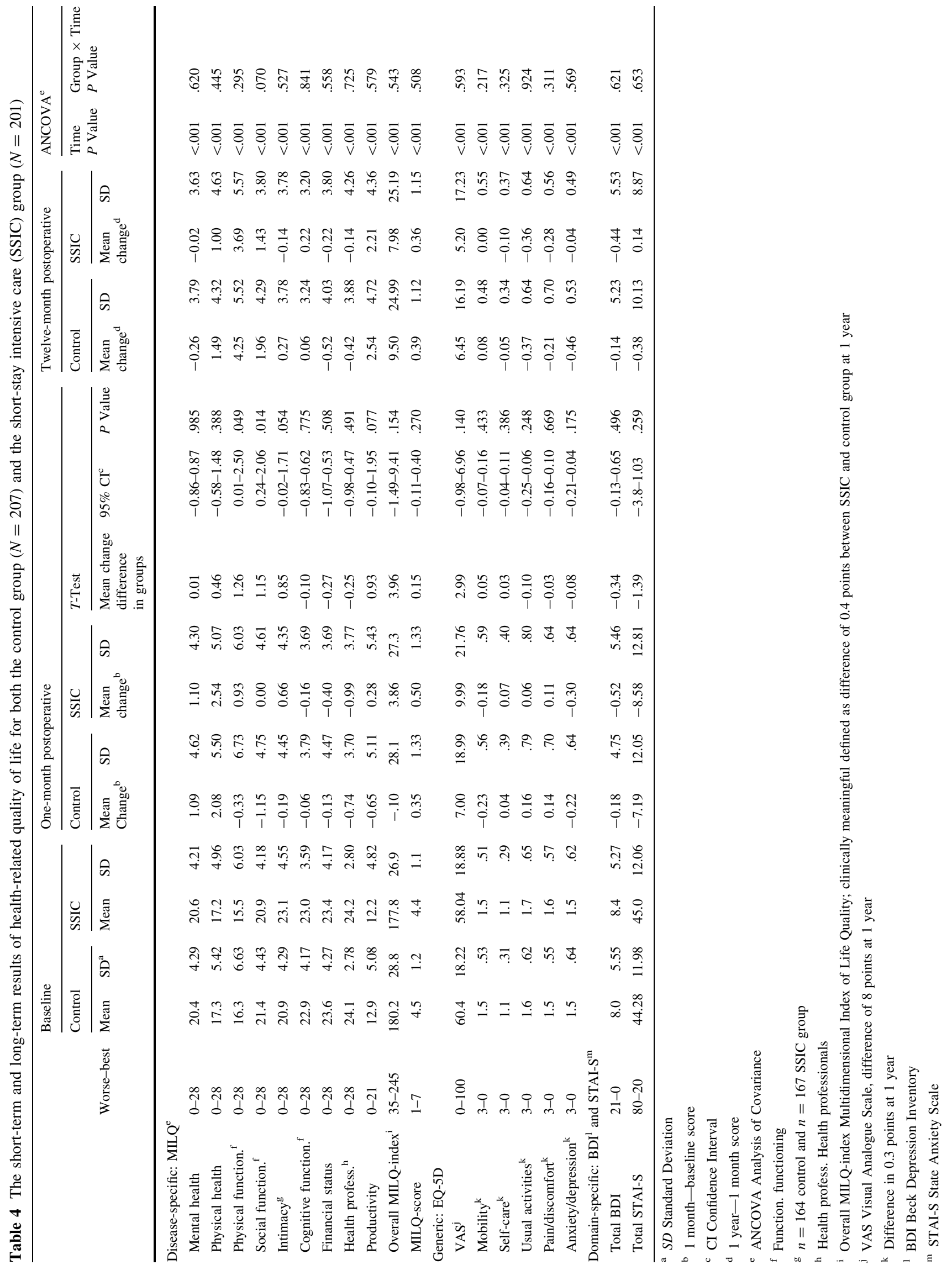


Table 5 Results of linear regression analysis for prediction models of HRQoL at 1 year after surgery

\begin{tabular}{|c|c|c|c|}
\hline & $\mathrm{B}$ & $95 \% \mathrm{CI}^{\mathrm{a}}$ & $P$ Value \\
\hline \multicolumn{4}{|c|}{ Model 1: Linear trend of MILQ-score ${ }^{\mathrm{b}}$} \\
\hline \multicolumn{4}{|c|}{$R$-square $=.334$} \\
\hline $\mathrm{B}_{\mathrm{o}}$ & 2.43 & 2.14 to 2.72 & $<.001$ \\
\hline Group & .024 & -.115 to .162 & .736 \\
\hline MILQ-score at baseline & -.435 & -.50 to -.38 & $<.001$ \\
\hline \multicolumn{4}{|c|}{ Model 2: Linear trend of Overall MILQ-Index ${ }^{\mathrm{b}}$} \\
\hline \multicolumn{4}{|c|}{$R$-square $=.208$} \\
\hline $\mathrm{B}_{\mathrm{o}}$ & 77.29 & & $<.001$ \\
\hline Group & -.148 & -3.64 to 3.35 & .934 \\
\hline Depression at baseline & -.406 & -.82 to .00 & .050 \\
\hline $\begin{array}{l}\text { Overall MILQ-Index at } \\
\text { baseline }\end{array}$ & -.368 & -.45 to -.29 & $<.001$ \\
\hline \multicolumn{4}{|c|}{ Model 3: Linear trend of EQ-5D VAS ${ }^{\mathrm{c}}$} \\
\hline \multicolumn{4}{|c|}{$R$-square $=.357$} \\
\hline $\mathrm{B}_{\mathrm{o}}$ & 30.33 & 24.2 to 36.4 & $<.001$ \\
\hline Group & -.648 & -.2 .81 to 1.51 & .556 \\
\hline Gender & -5.00 & -7.80 to -2.20 & $<.001$ \\
\hline Anxiety at baseline & .106 & .01 to .20 & .026 \\
\hline EQ-5D VAS at baseline & -.424 & -.48 to -.37 & $<.001$ \\
\hline
\end{tabular}

${ }^{a} C I$ Confidence Interval

b MILQ Multidimensional Index of Life Quality

c VAS Visual Analogue Scale

with cost containment as the main driving force [41]. Examples of these fast-track treatments that make IC discharge within a few hours after surgery possible are the use of fast-track anesthesia, normothermic temperature management, and early extubation at the intensive care. Due to the heterogeneity of the interventions, differences in casemix, various questionnaires and variable points in time used, a proper comparison between study findings difficult.

Nevertheless, our results confirm the finding of others [6, $12,13]$ that fast-track treatments do not have an impact on cognitive function. Royse et al. [15] found less posttraumatic stress and depression at 6-month postoperative in the fast-track group receiving low-dose anesthetics compared to the group which received a high dose. We did not find any evidence of a difference in the number of depressive and anxious feelings in the two groups in the first postoperative year. No comparison with other studies on the factor of pain can be made as these trials only reported on pain in the first days after the IC treatment [5, 10-14, 16, 17].

Clinical relevance of study findings

A strong point of our study is its sample size. In the current study, we were able to detect the possibility of a clinically meaningful QoL difference at 1 year (of 0.4 points on the
MILQ, of 0.3 points on the EQ-5D domains, and of 8 points on the EQ-5D VAS) between the SSIC and the control group. As none of these three scales at 1 year showed a statistically significant difference between both groups, the reported differences between both groups cannot be defined as a clinically meaningful. This indicates that the short-stay intensive care intervention does not have a relevant adverse impact on HRQoL at 1 year after surgery. In another study, we investigated the validity of both the MILQ and the EQ-5D. One of the findings was that the baseline ceiling effects (a high percentage of patients with a maximum score) in all EQ-5D dimensions were substantial, but these were not found on the MILQ-score and EQ-5D VAS. For the current study, this indicates that there is a possibility that not all relevant changes in HRQoL measured by means of the EQ-5D domains were identified. However, as outcomes reported by means of the EQ-5D domains are consistent with those of the EQ-5D VAS and the MILQ, the impact of this on the study conclusions is probably negligible.

The differences at 1-month postoperative on the physical and social functioning of the MILQ were significantly different between both groups, although this difference was no longer observed at long-term follow-up. As the power calculation was based on 1 year differences in HRQoL between the two groups, it is not possible to make a proper judgment whether the reported small positive effects in the SSIC group at short term are of clinical relevance. In a previous paper, we also reported generic HRQoL at 1 month after surgery as an outcome measure in the economic evaluation of the short-stay intensive care treatment. The patient's answers to the five EQ5D items were used to calculate the utility value, based on population weights [24] (ranging from 0 to 1), and subsequently the $\Delta$ quality adjusted life months ( $\Delta$ QALM's) was calculated. The generic HRQoL significantly improved in the SSIC group when compared to the control group. In the current study, this outcome was not reported because the aim of this paper was to report data from a patient's viewpoint and not from a so-called societal perspective. The observed difference measured at 1 month was very small (0.0238) and lower than the score of 0.03 which is considered clinically relevant to this study [43], [42].

If we compare our findings of the EQ-5D change scores of the EQ-5D at 12 months after surgery with those of results reported by Badia et al. in patients receiving scheduled surgery [22], the results of the domains of self-care, usual activities, pain/discomfort, and EQ-VAS are very similar, but the results of the other domains, such as mobility and anxiety/depression, are different. The mean score on mobility improved meaningfully clinical, whereas in our study population no such an improvement was observed. In contrast, patients in the SSIC study reported a decrease in the psychological dimension of the EQ-5D, whereas for patients scheduled for various types of surgery this score did not 
change. An explanation for these discrepancies between both studies can be related to the differences in patient characteristics. For instance, the mean age was lower in the study performed by Badia et al. compared to the mean age in our study. Furthermore, not only CABG patients but also liver transplant and other types of cardio surgical interventions were included in the Spanish study. Another explanation can be related to the differences in measuring the baseline QoL. The retrospective rating of the baseline QoL in the Spanish study can have resulted in an under- or overestimating of these scores and hence, in an under- or overestimating of the QoL change scores.

This study has the following limitations:

Ideally, preoperative administration of the HRQoL questionnaires should be done before emotional responses could confound the HRQoL measures, but this was not possible due to logistic reasons. However, as this study was comparative, this cannot have influenced the study findings much. A measurement of pain within a few days after treatment was not performed as it was practically impossible.

The generalizability of this study may be somewhat limited because the primary non-responders were older patients, and women were overrepresented compared to the responders. However, as all baseline characteristics for the responders and the non-responders of both treatment modalities were comparable, this cannot have a large impact on our study results.

Another limitation of this study is that standard hypothesis testing was used (the null hypothesis is equal means in groups). The recommended approach of Jones et al.
(1996), where conclusions are drawn on the basis of an appropriate confidence interval using the prespecified criteria of equivalence derived from the sample size calculation, was not done. Finally, fatigue of the responders may have biased the study findings. Other limitations concerning the study design and patient eligibility are described elsewhere [23].

\section{Conclusions and recommendations}

This randomized controlled equivalence trial evaluated the impact of a short-stay intensive care protocol on HRQoL in low-risk CABG patients. The short-stay intensive care treatment appears to have no effect on disease-specific and generic HRQoL in the first year after surgery. As none of the observed HRQoL differences between the two groups can be defined as clinically meaningful, while the safety and financial benefits of the SSIC intervention already have been demonstrated [23], from a patient's perspective short-stay intensive care can also be considered as a good alternative postoperative IC treatment in low-risk CABG patients.

Open Access This article is distributed under the terms of the Creative Commons Attribution Noncommercial License which permits any noncommercial use, distribution, and reproduction in any medium, provided the original author(s) and source are credited.

\section{Appendix}

See Table 6.

Table 6 Potential predictors of health-related quality of life used in the three regression models

\begin{tabular}{|c|c|c|c|}
\hline $\begin{array}{l}\text { Independent } \\
\text { Group }\end{array}$ & $\begin{array}{l}\text { Source } \\
\text { Case record form }\end{array}$ & $\begin{array}{l}\text { Coding } \\
\text { Care as usual }=0, \text { SSIC }=1\end{array}$ & Description \\
\hline \multicolumn{4}{|l|}{ Demographic data } \\
\hline Age (Years) & Database & Continuous & High age $[43-45]$ \\
\hline Female sex & Idem & Female $=1$, male $=0$ & Female $[43,45-49]$ \\
\hline Civil state & Self-reported & With partner $=1$, without partner $=0$ & No partner [48] \\
\hline Education & Idem & Primary school not finished yes $=1$, no $=0$ & Low education [26] \\
\hline \multicolumn{4}{|l|}{ Previous cardiac and medical history } \\
\hline $\begin{array}{l}\text { Left Ventricular ejection fraction } \\
>50 \% \\
<50 \%\end{array}$ & Database & $<50 \%=1,>50 \%=0$ & {$[43,50]$} \\
\hline $\begin{array}{l}\text { NYHA classification angina } \\
\text { I-II } \\
\text { III-IV }\end{array}$ & Idem & $\mathrm{III}-\mathrm{IV}=1, \mathrm{I}-\mathrm{II}=0$ & NYHA classification III-IV $[43,46]$ \\
\hline Hypertension & Idem & Yes $=1$, no $=0$ & History of Hypertension [45, 47, 49] \\
\hline $\mathrm{COPD}^{\mathrm{b}}$ & Idem & Yes $=1$, no $=0$ & History of COPD $[44,45,49]$ \\
\hline $\mathrm{CVA}^{\mathrm{c}}$ & Idem & Yes $=1$, no $=0$ & History of CVA $[45,49,52]$ \\
\hline
\end{tabular}


Table 6 continued

\begin{tabular}{|c|c|c|c|}
\hline $\begin{array}{l}\text { Independent } \\
\text { Group }\end{array}$ & $\begin{array}{l}\text { Source } \\
\text { Case record form }\end{array}$ & $\begin{array}{l}\text { Coding } \\
\text { Care as usual }=0, \text { SSIC }=1\end{array}$ & Description \\
\hline Smoking & Idem & Yes $=1$, no $=0$ & History of smoking [53] \\
\hline Diabetes mellitus & Idem & Yes $=1$, no $=0$ & Diabetes $[45,47,49]$ \\
\hline \multicolumn{4}{|l|}{ HRQoL Data ${ }^{\mathrm{d}}$} \\
\hline Baseline depression & Self-reported & & Depressive feelings at baseline $[26,53]$ \\
\hline Baseline anxiety & Idem & Continuous & Anxious feelings at baseline $[26,53]$ \\
\hline Baseline EQ-5D-VAS & Idem & Continuous & Baseline HRQoL $[45,49,54]$ \\
\hline Baseline MILQ-index & Idem & Continuous & Baseline HRQoL $[45,49,54]$ \\
\hline \multicolumn{4}{|l|}{ Other data } \\
\hline Postoperative (life) events ${ }^{\mathrm{e}}$ & Idem & Yes $=1$, no $=0$ & 'Stressful life event' $[43,51]$ \\
\hline \multicolumn{4}{|c|}{${ }^{\mathrm{a}}$ NYHA New York Heart Association } \\
\hline \multicolumn{4}{|c|}{ b $C O P D$ chronic obstructive pulmonary disease } \\
\hline \multicolumn{4}{|c|}{${ }^{\mathrm{c}}$ CVA Cerebrovascular accident } \\
\hline${ }^{\mathrm{d}} H R Q o L$ Heath-related quali & & & \\
\hline
\end{tabular}

\section{References}

1. Hawkes, C., Dhileepan, S \& Foxcroft, D. (2003). Early extubation for adult cardiac surgical patients. Cochrane database of systematic reviews. 4: p. CD003587.

2. Meade, M. O., et al. (2001). Trials comparing early vs late extubation following cardiovascular surgery. Chest, 120 (6 Sup$\mathrm{pl}), 445 \mathrm{~S}-453 \mathrm{~S}$.

3. Myles, P. S., et al. (2003). A systematic review of the safety and effectiveness of fast-track cardiac anesthesia. Anesthesiology, 99(4), 982-987.

4. Suter, P., Arma-Ganadis, A., \& Beaufils, F. (1994). Predicting outcome in ICU patients. 2nd European consensus conference in intensive care medicine. Intensive Care Medicine, 20(5), 390-397.

5. Bowler, I., et al. (2002). A combination of intrathecal morphine and remifentanil anesthesia for fast-track cardiac anesthesia and surgery. Journal of Cardiothoracic and Vascular Anesthesia, 16(6), 709-714.

6. Cheng, D. C., et al. (1996). Morbidity outcome in early versus conventional tracheal extubation after coronary artery bypass grafting: A prospective randomized controlled trial. Journal of Thoracic and Cardiovascular Surgery, 112(3), 755-764.

7. Cheng, D. C., et al. (1996). Early tracheal extubation after coronary artery bypass graft surgery reduces costs and improves resource use. A prospective, randomized, controlled trial. Anesthesiology, 85(6), 1300-1310.

8. Cheng, D. C., et al. (2001). The efficacy and resource utilization of remifentanil and fentanyl in fast-track coronary artery bypass graft surgery: A prospective randomized, double-blinded controlled, multi-center trial. Anesthesia and Analgesia, 92(5), 1094-1102.

9. Cheng, D. C., et al. (2003). Randomized assessment of resource use in fast-track cardiac surgery 1-year after hospital discharge. Anesthesiology, 98(3), 651-657.

10. Engoren, M., Luther, G., \& Fenn-Buderer, N. (2001). A comparison of fentanyl, sufentanil, and remifentanil for fast-track cardiac anesthesia. Anesthesia and Analgesia, 93(4), 859-864.

11. Fillinger, M. P., et al. (2002). Epidural anesthesia and analgesia: Effects on recovery from cardiac surgery. Journal of Cardiothoracic and Vascular Anesthesia, 16(1), 15-20.
12. Howie, M. B., et al. (2001). A randomized double-blinded multicenter comparison of remifentanil versus fentanyl when combined with isoflurane/propofol for early extubation in coronary artery bypass graft surgery. Anesthesia and Analgesia, 92(5), 1084-1093.

13. Kadoi, Y., et al. (2003). Comparative effects of propofol versus fentanyl on cerebral oxygenation state during normothermic cardiopulmonary bypass and postoperative cognitive dysfunction. Annals of Thoracic Surgery, 75(3), 840-846.

14. Mollhoff, T., et al. (2001). Comparative efficacy and safety of remifentanil and fentanyl in 'fast track' coronary artery bypass graft surgery: a randomized, double-blind study. British Journal of Anaesthesia, 87(5), 718-726.

15. Royse, C., et al. (2003). Prospective randomized trial of high thoracic epidural analgesia for coronary artery bypass surgery. Annals of Thoracic Surgery, 75(1), 93-100.

16. Shroff, A., Rooke, G. A., \& Bishop, M. J. (1997). Effects of intrathecal opioid on extubation time, analgesia, and intensive care unit stay following coronary artery bypass grafting. Journal of Clinical Anesthesia, 9(5), 415-419.

17. Zarate, E., et al. (2000). Fast-track cardiac anesthesia: use of remifentanil combined with intrathecal morphine as an alternative to sufentanil during desflurane anesthesia. Anesthesia and Analgesia, 91(2), 283-287.

18. Heyland, D. K., et al. (1998). Frequency and methodologic rigor of quality-of-life assessments in the critical care literature. Critical Care Medicine, 26(3), 591-598.

19. Mayou, R., \& Bryant, B. (1993). Quality of life in cardiovascular disease. British Heart Journal, 69(5), 460-466.

20. Nowels, D., et al. (2005). Validation of the EQ-5D quality of life instrument in patients after myocardial infarction. Quality of Life Research, 14(1), 95-105.

21. Schweikert, B., Hahmann, H., \& Leidl, R. (2006). Validation of the EuroQol questionnaire in cardiac rehabilitation. Heart, 92(1), 62-67.

22. Badia, X., et al. (2001). Using the EuroQol-5D to measure changes in quality of life 12 months after discharge from an intensive care unit. Intensive Care Medicine, 27(12), 1901-1907.

23. van Mastrigt, G. A., et al. (2006). Short-stay intensive care after coronary artery bypass surgery: randomized clinical trial on safety and cost-effectiveness. Critical Care Medicine, 34(1), 65-75. 
24. Dolan, P. (1997). Modeling valuations for EuroQol health states. Medical Care, 35(11), 1095-1108.

25. Avis, N. E., et al. (1996). Development of the multidimensional index of life quality. A quality of life measure for cardiovascular disease. Medical Care, 34(11), 1102-1120.

26. Duits, A. A., et al. (1997). Prediction of quality of life after coronary artery bypass graft surgery: a review and evaluation of multiple, recent studies. Psychosomatic Medicine, 59(3), 257-268.

27. Hurel, D., et al. (1997). Quality of life 6 months after intensive care: results of a prospective multicenter study using a generic health status scale and a satisfaction scale. Intensive Care Medicine, 23(3), 331-337.

28. Cuthbertson, B. H., et al. (2005). Quality of life before and after intensive care. Anaesthesia, 60(4), 332-339.

29. Konopad, E., et al. (1995). Quality of life measures before and 1 year after admission to an intensive care unit. Critical Care Medicine, 23(10), 1653-1659.

30. Parsonnet, V., Dean, D., \& Bernstein, A. D. (1989). A method of uniform stratification of risk for evaluating the results of surgery in acquired adult heart disease. Circulation, 79(6 Pt 2), 12-13.

31. Definitions of terms of the society of Thoracic surgeons national cardiac surgery database: http://www.sts.org. Series Definitions of terms of the society of Thoracic surgeons national cardiac surgery database: http://www.sts.org [cited; Available from: http://www.sts.org.

32. Falger, P. S., \& EHWJ van Leuteren, K. S. J. (2000). Wederzijdse beinvloeding van de kwaliteit van leven van patienten en significante anderen na een hartinfarct of een 'coronary artery bypass-graft'-operatie. Gedrag en Gezondheid, 28(5), 274-287.

33. EuroQoL-Group. (1990). EuroQoL-a new facility for the measurement of health-related quality of life. Health Policy, 16(3), 199-208.

34. Beck, A. T., \& Steer, R. A. (1987). Beck depression inventory manual (pp. 561-571). Toronto: Hatcourt, Brace, Jovanovich.

35. van der Ploeg, H. D., PB. Spielberger, C.(1986). Manual for the State-Trait Anxiety Inventory (STAI). Lisse: Swets and Zeitlinger BV.

36. Eisenberg, E., et al. (2001). Prevalence and characteristics of post coronary artery bypass graft surgery pain (PCP). Pain, 92(1-2), $11-17$.

37. Boudrez, H., \& De Backer, G. (2001). Psychological status and the role of coping style after coronary artery bypass graft surgery. Results of a prospective study. Quality of Life Research, 10(1), 37-47.

38. Langeluddecke, P., et al. (1989). A prospective evaluation of the psychosocial effects of coronary artery bypass surgery. Journal of Psychosomatic Research, 33(1), 37-45.

39. Staquet, M., et al. (1996). Guidelines for reporting results of quality of life assessments in clinical trials. Quality of Life Research, 5(5), 496-502.
40. Johnson, R. G. (2006). Grading the evidence for shortened intensive care unit stays after cardiac operations. Critical Care Medicine, 34(1), 242-243.

41. Higgins, T. L. (1992). Pro: early endotracheal extubation is preferable to late extubation in patients following coronary artery surgery. Journal of Cardiothoracic and Vascular Anesthesia, 6(4), 488-493.

42. Marra, C. A., et al. (2005). A comparison of generic, indirect utility measures (the HUI2, HUI3, SF-6D, and the EQ-5D) and disease-specific instruments (the RAQoL and the HAQ) in rheumatoid arthritis. Social Science and Medicine, 60(7), 15711582.

43. Chocron, S., et al. (1996). Prospective study of quality of life before and after open heart operations. Annals of Thoracic Surgery, 61(1), 153-157.

44. Welke, K. F., et al. (2003). Patient characteristics can predict improvement in functional health after elective coronary artery bypass grafting. Annals of Thoracic Surgery, 75(6), 1849-1855. (discussion 1855).

45. Herlitz, J., et al. (2005). Determinants for an impaired quality of life 10 years after coronary artery bypass surgery. International Journal of Cardiology, 98(3), 447-452.

46. Simchen, E., et al. (2001). Sociodemographic and clinical factors associated with low quality of life 1 year after coronary bypass operations: the Israeli coronary artery bypass study (ISCAB). Journal of Thoracic and Cardiovascular Surgery, 121(5), 909919.

47. Yun, K. L., et al. (1999). Time related quality of life after elective cardiac operation. Annals of Thoracic Surgery, 68(4), 1314-1320.

48. Phillips Bute, B., et al. (2003). Female gender is associated with impaired quality of life 1 year after coronary artery bypass surgery. Psychosomatic Medicine, 65(6), 944-951.

49. Herlitz, J., et al. (1999). Determinants of an impaired quality of life 5 years after coronary artery bypass surgery. Heart, 81(4), 342-346.

50. Caine, N., Sharples, L. D., \& Wallwork, J. (1999). Prospective study of health related quality of life before and after coronary artery bypass grafting: outcome at 5 years. Heart, 81(4), 347-351.

51. Pirraglia, P. A., et al. (1999). Depressive symptomatology in coronary artery bypass graft surgery patients. International Journal of Geriatric Psychiatry, 14(8), 668-680.

52. Al-Ruzzeh, S., et al. (2005). Predictors of poor mid-term health related quality of life after primary isolated coronary artery bypass grafting surgery. Heart, 91(12), 1557-1562.

53. Schrader, G., et al. (2004). Predictors of depression 3 months after cardiac hospitalization. Psychosomatic Medicine, 66(4), 514-520.

54. Rumsfeld, J. S., et al. (2001). Changes in health-related quality of life following coronary artery bypass graft surgery. Annals of Thoracic Surgery, 72(6), 2026-2032. 